\title{
Uma Investigação sobre Disfunções Familiares em Estudantes de Medicina
}

\author{
An investigation into Familiar Dysfunctions \\ among Medical Students
}

\author{
Isabelle Christine de Moraes Motta ${ }^{1 \oplus}$ \\ Rita de Cássia Menezes Soares ${ }^{I}$ \\ Terezinha de Souza Agra Belmonte
}

\section{PALAVRAS-CHAVE}

- Estudantes de Medicina.

- Saúde Mental.

- Transtornos Mentais.

- Grupo Social.

- Educação Médica.
Introdução: As Diretrizes Curriculares Nacionais do Curso de Graduação em Medicina (2014) determinam como perfil do egresso médico uma identidade generalista, humanista, crítica, reflexiva e multicultural. Isto exige habilidades biopsicomotoras qualificadas (competências de cuidar, curar e reabilitar pessoas e suas famílias). Os núcleos psicopedagógicos nacionais encontram no atendimento desses estudantes conflitos pessoais, pedagógicos e familiares traduzidos em transtornos mentais. As disfunções familiares estarão relacionadas ao aumento dessa prevalência na graduação médica (socialização secundária)? Uma socialização primária insatisfatória contribuiria para o adoecer no processo do adultecer? Objetivos: Verificar o perfil sociodemográfico, psiquiátrico e familiar de estudantes de Medicina numa instituição federal do Estado do Rio de Janeiro, Brasil. Métodos: A pesquisa foi um estudo quali-quantitativo, com intervenção observacional, exploratória, transversal, descritiva e inferencial. A coleta de dados foi feita por meio de dois instrumentos: (1) um questionário construído para esta pesquisa, autoaplicável, com variáveis sociopsicodemográficas e categorias familiares disfuncionais descritas na literatura; (2) o Mini International Neuropsychiatric Interview (Mini), versão 5.0.0, compatível com os critérios do DSM-IV e validado por Patrícia Amorim/2000. Os dados foram colhidos após consentimento livre e esclarecido (TCLE) e organizados em banco de dados, no Excel. A análise foi feita com o software estatístico R. As variáveis quantitativas foram analisadas por desvio padrão, média, mediana e moda. As variáveis qualitativas tiveram análises de frequência. Foram realizados testes estatísticos: exato de Fisher, Wilcoxon bilateral, Qui-Quadrado e Shapiro-Wilk, de acordo com as variáveis e objetivos da análise. A pesquisa foi aprovada pelo Comitê de Ética e Pesquisa. CAAE: 67590317.5.0000.5258, em 25/07/2017. Resultados: Foram avaliados 129 alunos, com 18 a 32 anos de idade, do primeiro ao 12 - período da graduação, $49 \%$ do sexo feminino e $51 \%$ do sexo masculino. Prevalência de transtorno de ansiedade generalizada: 39,53\%; de depressão (atual ou recorrente): 32,56\%; e risco de suicídio: 28,68\%. A depressão possuiu forte correlação com história familiar de transtorno psiquiátrico (p-valor: 7.639e08) e com o relacionamento dos pais. Ela esteve presente naqueles com pais divorciados, viúvos ou sem relacionamento (p-valor: 0.008291). O risco de suicídio foi maior entre acadêmicos do ciclo básico, do primeiro ao quarto período da graduação ( $p$-valor: 0,01), e possuía forte correlação com ter sofrido ou sofrer bullying ( $p$-valor: 0,02), ter uma religião (p-valor: 0,006), e depressão ( $p$-valor: 0,03) ou transtorno de pânico (p-valor: 4.903e-05). Houve correlação entre depressão ( $p$-valor: 1.248 e-09) e risco de suicídio ( $p$ valor: 0.0009) nos que tinham problemas de comunicação na família. Transtorno de pânico foi observado em 17,05\% dos entrevistados, sexo feminino (p-valor: 0.00583). Isto decorre do relacionamento dos pais. Os alunos com pais casados foram os mais afetados (p-valor: 0.01284) Conclusões: Os dados colhidos demonstraram correlações entre disfunções familiares e transtornos psiquiátricos, como depressão, risco de suicídio, transtorno de pânico e transtorno de ansiedade generalizada. Existe a necessidade de um ambiente de ensino-aprendizagem com espaços e cenários e educadores médicos que identifiquem precocemente o sofrimento psíquico na socialização secundária do futuro médico. 


\section{KEY-WORDS}

- Medical Students.

- Mental Health.

- Mental Disorders.

- Social Group.

- Medical Education.

Recebido em 9/5/19

Aceito em 29/6/19

\section{INTRODUÇÃO}

Nas culturas primitivas, o sacerdote e o curandeiro (o xamã) tinham a sua personalidade e a adequada capacitação pelos antecessores para lidar com as tribos, sua cultura e diferenças como um instrumento importante para ser escolhido para seu ofício ${ }^{1}$.
ABSTRACT

Introduction: The National Curricular Guidelines for Undergraduate Medicine Courses (2014) determine the profile of medical graduates as having a generalist, humanistic, critical, reflective and multicultural identity. This requires qualified biopsychomotor skills (skills of caring, healing and rehabilitating people and their families). The national psycho-pedagogical support centres find in these students personal, pedagogical and family conflicts translated into mental disorders. Will family dysfunctions be related to increased prevalence among medical undergraduate students (secondary socialization)? Would unsatisfactory primary socialization contribute to becoming ill in the ageing process? Objectives: To verify the socio-demographic, psychiatric and family profile of medical students in a federal institution in the state of Rio de Janeiro, Brazil. Methods: The research was a qualitative-quantitative study, involving an observational intervention, and of an exploratory, transversal, descriptive and inferential nature. Data collection was performed through two strategies: (1) a self-administered questionnaire built for this research, with socio-psychodemographic variables and dysfunctional family categories described in the literature. (2) the Mini International Neuropsychiatric Interview (Mini), 5.0 .0 version, compatible with the DSM-IV criteria; Validated by Patrícia Amorim/2000. Data were collected after an Informed Consent Form (ICF) was signed and a database was created in Excel. The analysis was performed with the statistical software R. Quantitative variables were analysed by standard deviation, mean, median and mode. The qualitative variables underwent frequency analyses. Statistical tests were performed by Fisher exact test, bilateral Wilcoxon, chi-Square and Shapiro-Wilk, according to the variables and objectives of the analysis. The research was approved by the Ethics and Research Committee under number CAAE: 67590317.5.0000.5258, on 25/07/2017. Results: 129 students were evaluated, aged 18 to 32 years, ranging from the 1st to 12th semester of the undergraduate course, $49 \%$ were female and $51 \%$ male. The prevalence of generalized anxiety disorder was $39.53 \%$; depression (current or recurrent), $32.56 \%$ and suicide risk, $28.68 \%$. Depression had a strong correlation with a family history of psychiatric disorder (p-value: $7.639 \mathrm{e}-08$ ) and the parents' relationship. It was present in those with divorced parents, widowed or without a relationship ( $P$ value: 0.008291 ). The risk of suicide was higher among students of the basic cycle, 1st to 4th semester of the course ( $p$-value: 0.01 ), that had a strong correlation with suffering or still suffer bullying ( $p$-value: 0.02), having a religion (p-value: 0.006 ), and depression (p-value: 0.03 ) or panic disorder (p-value: $4.903 e-05$ ). There was a correlation between depression (p-value: 1.248 and-09) and suicide risk (p-value: 0.0009) in those who had communication problems in the family. Panic disorder was observed in $17.05 \%$ of the interviewees, females ( $p$-value: 0.00583). This stems from the parents' relationship. Students with married parents were the most affected. (p-value: 0.01284) Conclusions: The collected data showed correlations between family dysfunctions and psychiatric disorders such as depression, suicide risk, panic disorder, and generalized anxiety disorder. There is a need for a teaching/learning environment, including adequate spaces and scenarios, and also medical educators who can, at an early stage, identify psychological distress in the secondary socialization of the future physician. 
se por uma formação saudável do estudante de Medicina até se tornar um egresso².

A psicopatologia do estudante de Medicina começou a ser pesquisada e estruturada de forma sistemática a partir do século $X^{3}$. O professor Gaudino Loreto, da Universidade Federal de Pernambuco, ofereceu assistência psiquiátrica a estudantes de Medicina em 1957. Ele publicou uma tese sobre a sua experiência. Outros centros nacionais tentaram criar esses serviços, mas as interferências da política universitária, a falta de um setting para o atendimento dos alunos e a carência de recursos enfraqueceram o processo ${ }^{3}$.

Uma das contribuições marcantes para a reflexão sobre o tema foi o livro O Universo Psicológico do Futuro Médico ${ }^{4}$. Ele apresentou as complexidades do rito de passagem do estudante de Medicina desde o seu ingresso, vocação e trajetória do ciclo básico ao ciclo clínico até o internato. A configuração dos principais núcleos de apoio psicopedagógico nacional contribuiu para as pesquisas na informação e formação do contexto da educação médica ${ }^{4}$.

"A Carta de Marília" foi um documento referendado no XI Fórum dos Serviços de Apoio ao estudante de Medicina, no $54^{\circ}$ Congresso Paulista de Educação Médica, na cidade de Brasília (Forsa Cobem/13 de outubro de 2016). Ele declara que o cuidado da saúde emocional e psíquica do estudante deve ser considerado objetivo da formação médica, sendo responsabilidade da instituição a garantia de assistência à saúde mental do corpo discente ${ }^{5,6}$.

Um estudo com a Plataforma Veras, em 22 escolas médicas, demonstrou que o ambiente de ensino numa faculdade de Medicina influencia o bem-estar e a qualidade de vida, principalmente em estudantes do sexo feminino e nos matriculados no final do curso ${ }^{7}$.

Uma revisão sobre o conteúdo do tema "núcleos psicopedagógicos em medicina" revela que, no atendimento a esses estudantes, a prevalência é de transtornos psiquiátricos e psicológicos decorrentes de conflitos pessoais, pedagógicos e familiares. Os artigos destacam os transtornos de ansiedade, depressão e suicídio. Essa situação é correlacionada ao excesso de conteúdo a ser aprendido em pouco tempo, à cobrança familiar de que "eles, alunos já são capazes de atuarem como médicos" ou ainda à sensação de culpa pelo afastamento do núcleo familiar para dedicar-se aos estudos s,4,8-14 $^{\text {. }}$

A socialização secundária (formação universitária) na sociedade contemporânea é imprescindível nas habilidades e competências do médico clínico geral e de família para um estado de saúde de completo bem-estar físico, mental e social ${ }^{4,11,15-17}$.

As novas organizações familiares podem ser um fator determinante da má socialização primária e consequente adoe- cimento da criança, adolescente e jovem ${ }^{17,18}$. A família é fonte de suporte, mas também pode ser geradora de estresse e está envolvida no processo saúde-doença ${ }^{19}$. Ela "ensina a criança a aprender"18,19.

O processo de socialização consiste em duas fases: a primária, fase que o indivíduo experimenta na infância e em virtude da qual se torna membro da sociedade. Isto acontece no seio familiar, em que a carga afetiva é importante, e nela se aprende a transmissão de conteúdo do mundo adulto; e a secundária, fase posterior, fase cognitiva que incorpora o indivíduo no mundo objetivo social ${ }^{19}$

O conceito de família disfuncional baseia-se numa compreensão da psiquiatria psicodinâmica, dos aspectos vinculares e do diagnóstico situacional e tratamento subsequente. Os sintomas do indivíduo são analisados no contexto das relações com outras pessoas e grupos, e não como problemas individuais de causa e efeito. Esse termo indica a existência de famílias em que o relacionamento entre seus membros não é equilibrado. O resultado pode ser traduzido em problemas como introversão, transtornos psicológicos, psiquiátricos (depressão e transtorno de ansiedade generalizada) e problemas de baixa autoestima ${ }^{17,18,20}$.

Emoções suprimidas, limites frágeis, relacionamento abusivo, problemas de comunicação com padrões de grande conflitualidade - desinteresse pelos assuntos do outro, ausência de diálogo, divergências de opiniões, compreensão e crenças -, regras rígidas e inconsistentes, restrição de sentimentos e autoexpressão, abuso de álcool, drogas e medicamentos e alienação parental são as categorias que a literatura revela como sendo as envolvidas nas disfunções familiares ${ }^{17,18,20}$.

Uma família estruturada é imprescindível no momento em que qualquer estudante vivencia a trajetória de superação dos desafios da sua identidade profissional ${ }^{20}$.

As Diretrizes Curriculares Nacionais do Curso de Graduação em Medicina (2014) determinam a necessidade do egresso médico com uma identidade profissional, generalista, humanista, crítica, reflexiva e multicultural para atuação nas diversas áreas de atenção à saúde ${ }^{15,16}$. Essas diretrizes encontram resistências para serem implantadas nas escolas médicas devido à diversidade docente, capacitada no modelo biomédico em detrimento do humanístico. A família acadêmica encontra-se em processo de transição, assim como a família nuclear dos seres humanos.

O objetivo desta pesquisa é verificar a relação entre o perfil sociodemográfico, psiquiátrico e familiar de um grupo de estudantes de Medicina de uma universidade federal no Rio de Janeiro e observar se os problemas psiquiátricos já atribuídos a essa população estariam correlacionados à presença de disfunções familiares ${ }^{8-10,17,18,20,22}$. 


\section{MÉTODO}

Foi realizado um estudo quali-quantitativo, com intervenção observacional (levantamento de características do campo da pesquisa), exploratória, transversal, descritiva e inferencial. A coleta de dados foi feita por meio de dois instrumentos: (1) um questionário construído para esta pesquisa, autoaplicável, com variáveis psicossociobiodemográficas, e as categorias familiares disfuncionais ${ }^{18}$; (2) o Mini International Neuropsychiatric Interview (Mini), versão 5.0.0, validado por Patrícia Amorim $/ 2000^{23}$, que consiste numa entrevista diagnóstica padronizada breve (15-30 minutos), compatível com os critérios do DSM-IV. O Mini é destinado à utilização na prática clínica, na pesquisa em atenção primária e em psiquiatria. Pode ser utilizado por clínicos após rápido treinamento (de uma a três horas).

A pesquisa foi aprovada pelo Comitê de Ética e Pesquisa (CEP) da Instituição. CAAE: 67590317.5.0000.5258, em $25 / 07 / 2017$. Os voluntários eram convidados a participar da pesquisa após a sua divulgação em salas de aula e redes sociais. Os interessados, após assinatura do Termo de Consentimento Livre e Esclarecido, respondiam aos questionários. As informações coletadas foram anônimas. Foi realizada estatística descritiva e análise exploratória dos dados. Elas foram organizadas em um banco de dados, em Excel. Para variáveis quantitativas foram analisadas medidas-resumo, como desvio padrão, média, mediana e moda. Para variáveis qualitativas foram feitas análises de frequência. A análise inferencial foi executada com auxílio do software R. Foram realizados os seguintes testes estatísticos: teste exato de Fisher, teste de Wilcoxon bilateral, teste Qui-Quadrado e teste de Shapiro-Wilk, de acordo com as variáveis analisadas e objetivos da análise.

\section{RESULTADOS}

\section{Dados sociodemográficos}

Participaram da pesquisa 129 alunos do primeiro ao $12^{\circ}$ período, $49 \%$ do sexo feminino e $51 \%$ do sexo masculino, com idades entre 18 e 32 anos. Oitenta e sete por cento dos alunos eram naturais do Rio de Janeiro (Tabela 1); 48,06\% dos alunos pertenciam ao ciclo básico do curso (primeiro ao quarto período), 34,89\% ao ciclo clínico (quinto ao oitavo período) e $17,05 \%$ ao internato (nono ao 12 o período); $36,43 \%$ eram católicos, $0,78 \%$ umbanda, $3,88 \%$ espíritas, $22 \%$ protestantes, $0,78 \%$ judeu, $41,09 \%$ ateus ou sem religião; $51,94 \%$ dos entrevistados estavam namorando, 2,33\% eram casados e $45,74 \%$ solteiros; $66,77 \%$ dos alunos referiam que os pais eram casados, 20,93\% que os pais eram divorciados, $6,20 \%$ deles moravam juntos, mas não eram casados, 2,33\% com pai ou mãe viúvos e 3,10\% desses pais não possuíam relacionamento afetivo; $57,30 \%$ dos entrevistados habitavam com os pais atualmente, sendo que $19,38 \%$ viviam com a mãe, $6,20 \%$ com outros parentes, $5 \%$ com o parceiro ou marido, $3,10 \%$ moravam sozinhos e $8,53 \%$ com outras pessoas, que não eram da família; $31,01 \%$ dos estudantes relataram ter mudado de residência após a entrada no curso de Medicina e, assim, deixaram de morar com pessoas da família, sendo que $85 \%$ referiam impactos negativos em suas vidas com essa mudança; $76,74 \%$ dos estudantes possuíam irmãos; $55,04 \%$ dos entrevistados referiram ter escolhido a graduação em Medicina por vocação, 9,30\% por influência familiar, $33,33 \%$ por status profissional e financeiro e $2,33 \%$ por outros motivos; $25,58 \%$ dos estudantes disseram desejar cursar Medicina desde crianças, 52,71\% optaram por Medicina quando adolescentes e $21,71 \%$ quando adultos; $58,91 \%$ dos acadêmicos tentaram vestibular para Medicina mais de uma vez; $37,98 \%$ ingressaram na universidade pelo sistema de cotas; $10,85 \%$ dos voluntários possuíam formação superior prévia à Medicina; 65,89\% (85 alunos) referiram ter sofrido bullying em algum momento de suas vidas, $82,35 \%$ revelaram ter sido na família e $97,65 \%$ na escola; $8,24 \%$ dos 85 alunos narraram a continuidade do sofrimento de bullying na faculdade; $28,68 \%$ (37 alunos) apresentaram alguma queixa relacionada à família, como desinteresse pelos assuntos dos outros membros da família; 35,66\% relataram ter família rígida e $85,16 \%$ possuíam boa relação familiar; $13,28 \%$ sofriam com problemas de vício na família (principalmente etilismo); 8,59\% possuíam algum membro familiar violento; $34,37 \%$ possuíam história familiar positiva para doença psiquiátrica, como depressão, transtorno bipolar, transtorno de pânico e transtorno de ansiedade generalizada; 47,29\% referiram ter problemas de comunicação, como ausência de diálogo.

\section{Transtornos psiquiátricos}

Sofriam de depressão (atual ou recorrente) 32,56\% dos estudantes; $39,53 \%$ sofriam com transtorno de ansiedade generalizada; $28,68 \%$ apresentavam risco de suicídio (alto, médio ou baixo); 3,10\% possuíam transtorno distímico atual; 0,78\% apresentaram episódio hipomaníaco atual; 2,33\% apresentaram episódio maníaco atual; 2,33\% apresentaram episódio maníaco passado; $17,05 \%$ dos entrevistados manifestaram transtorno de pânico na vida inteira; 0,78\%, transtorno de pânico atual; 2,33\% apresentaram agorafobia; 4,65\%, fobia social; $3,88 \%$, transtorno obsessivo compulsivo; $1,55 \%$ possuíam compulsões; $1,55 \%$ sofriam com transtorno de estresse pós-traumático; $0,78 \%$ apresentaram bulimia nervosa. Nessa amostra, não foi encontrada anorexia nervosa, síndrome psicótica, transtorno de personalidade antissocial, dependência ou abuso de álcool e outras drogas (Tabela 2). 


\begin{tabular}{|c|c|c|}
\hline \multicolumn{3}{|c|}{$\begin{array}{c}\text { TABELA } 1 \\
\text { Dados sociodemográficos }\end{array}$} \\
\hline Variável & $\mathbf{N}$ & $\%$ \\
\hline \multicolumn{3}{|l|}{ Faixa etária (anos) } \\
\hline 18 a 23 & 76 & 58,92 \\
\hline 24 a 29 & 49 & 37,98 \\
\hline 30 ou mais & 4 & 3,10 \\
\hline \multicolumn{3}{|l|}{ Sexo } \\
\hline Feminino & 64 & 49,61 \\
\hline Masculino & 65 & 50,39 \\
\hline \multicolumn{3}{|l|}{ Período } \\
\hline 1 a 4 (ciclo básico) & 62 & 48,06 \\
\hline 5 a 8 (ciclo clínico) & 45 & 34,88 \\
\hline 9 a 12 (internato) & 22 & 17,05 \\
\hline \multicolumn{3}{|l|}{ Relacionamento } \\
\hline Solteiro & 59 & 45,74 \\
\hline Namorando & 67 & 51,94 \\
\hline Casado & 3 & 2,33 \\
\hline \multicolumn{3}{|l|}{ Naturalidade } \\
\hline RJ & 113 & 87,60 \\
\hline Outras & 16 & 12,40 \\
\hline \multicolumn{3}{|l|}{ Com quem mora } \\
\hline Sozinho & 4 & 3,10 \\
\hline Pais & 74 & 57,36 \\
\hline Mãe & 25 & 19,38 \\
\hline Outros parentes & 10 & 7,75 \\
\hline Parceiro & 5 & 3,88 \\
\hline Outras pessoas & 11 & 8,53 \\
\hline \multicolumn{3}{|c|}{ Mudou de residência ao entrar no curso } \\
\hline Sim & 40 & 31,01 \\
\hline Não & 89 & 68,99 \\
\hline \multicolumn{3}{|l|}{ Impacto da mudança } \\
\hline Positivo & 4 & 10,00 \\
\hline Negativo & 34 & 85,00 \\
\hline Nenhum ou não respondeu & 2 & 5,00 \\
\hline \multicolumn{3}{|l|}{ Motivo da escolha do curso } \\
\hline Status financeiro ou profissional & 43 & 33,33 \\
\hline Influência familiar & 12 & 9,30 \\
\hline Vocação & 71 & 55,04 \\
\hline Outros & 3 & 2,33 \\
\hline \multicolumn{3}{|l|}{ Momento da escolha do curso } \\
\hline Infância & 33 & 25,58 \\
\hline Adolescência & 68 & 52,71 \\
\hline Adultecer & 28 & 21,71 \\
\hline \multicolumn{3}{|l|}{ Sofreu bullying } \\
\hline Sim & 85 & 65,89 \\
\hline Na famíla & 70 & 82,35 \\
\hline Na escola & 83 & 97,65 \\
\hline Não & 44 & 34,11 \\
\hline \multicolumn{3}{|l|}{ Ingressou por cotas } \\
\hline Sim & 49 & 37,98 \\
\hline Não & 80 & 62,02 \\
\hline \multicolumn{3}{|l|}{ Religião } \\
\hline Católica & 47 & 36,43 \\
\hline Evangélica & 22 & 17,05 \\
\hline Ateu ou sem religião & 53 & 41,09 \\
\hline Outras & 7 & 5,43 \\
\hline
\end{tabular}

\begin{tabular}{|c|c|c|}
\hline \multicolumn{3}{|c|}{$\begin{array}{c}\text { TABELA } 2 \\
\text { Dados familiares }\end{array}$} \\
\hline Variável & $\mathbf{N}$ & $\%$ \\
\hline \multicolumn{3}{|l|}{ Queixa na família } \\
\hline Sim & 37 & 28,68 \\
\hline Não & 92 & 71,32 \\
\hline \multicolumn{3}{|l|}{ Relacionamento dos pais } \\
\hline Casados & 87 & 67,44 \\
\hline Divorciados & 27 & 20,93 \\
\hline Pai ou mãe viúvos & 3 & 2,33 \\
\hline Outro & 12 & 9,30 \\
\hline \multicolumn{3}{|l|}{ Família funcional } \\
\hline Sim & 112 & 87,50 \\
\hline Não & 16 & 12,50 \\
\hline \multicolumn{3}{|c|}{ Problemas de comunicação } \\
\hline Sim & 61 & 47,29 \\
\hline Não & 68 & 52,71 \\
\hline \multicolumn{3}{|l|}{ Membro violento } \\
\hline Sim & 11 & 8,59 \\
\hline Não & 117 & 91,41 \\
\hline \multicolumn{3}{|l|}{ Membro com vícios } \\
\hline Sim & 17 & 13,28 \\
\hline Não & 111 & 86,72 \\
\hline \multicolumn{3}{|c|}{ História de doença psiquiátrica } \\
\hline Sim & 44 & 34,37 \\
\hline Não & 84 & 65,63 \\
\hline \multicolumn{3}{|l|}{ Família rígida } \\
\hline Sim & 46 & 35,66 \\
\hline Não & 83 & 64,34 \\
\hline \multicolumn{3}{|l|}{ Boa relação familiar } \\
\hline Sim & 109 & 85,16 \\
\hline Não & 19 & 14,84 \\
\hline
\end{tabular}

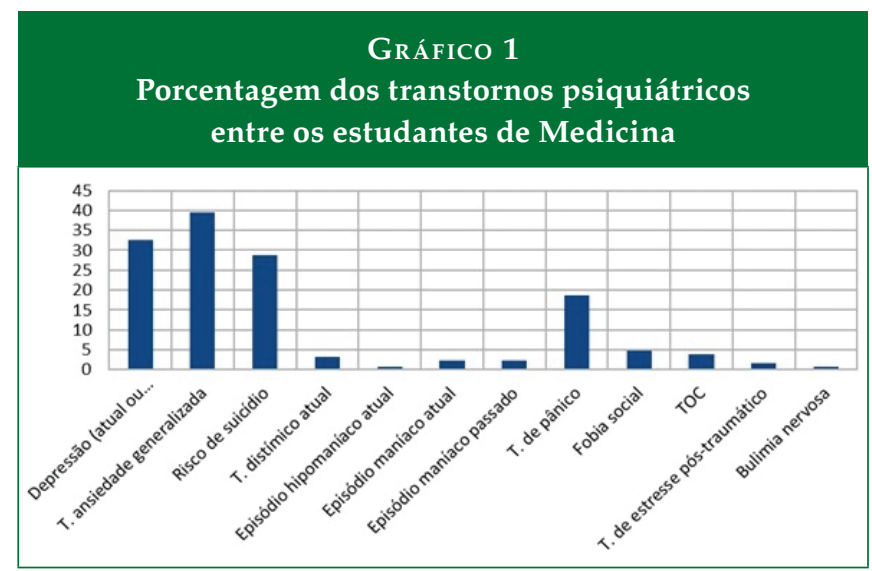


TABELA 3

\section{Cruzamento de variáveis, testes estatísticos realizados e resultados encontrados}

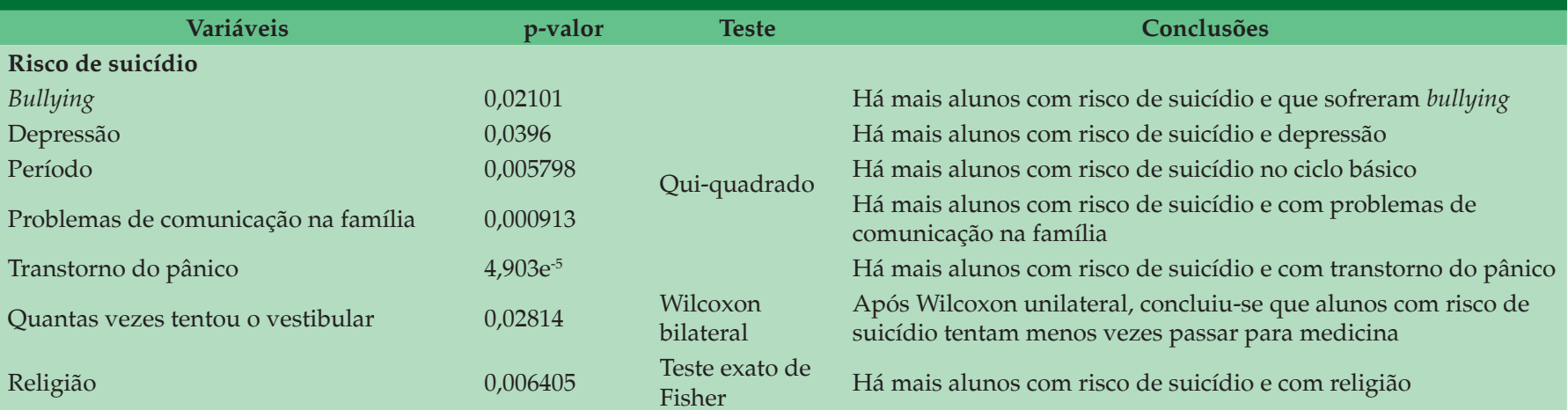

Histórico familiar de doença psiquiátrica

Depressão

Transtorno de ansiedade generalizada

Transtorno de ansiedade generalizada

$7,639 \mathrm{e}^{-8} \quad$ Qui

0,000457 quadrado

Há mais alunos com histórico familiar e com depressão

Há menos alunos com histórico familiar e com transtorno
Bullying

Depressão

Mudança de residência

Período

0,004967

0,000223

0,0236

0,01052

Problemas de comunicação na família

0,02738

0,02692

Quando decidiu fazer medicina

\section{Depressão}

Problemas de comunicação na família

$1,248 \mathrm{e}^{-9}$

Relacionamento dos pais

0,01588

\section{Transtorno do pânico}

Bullying

0,01604

Quando decidiu fazer medicina

$1,332 \mathrm{e}^{-6}$

Relacionamento dos pais

0,009363

Quantas vezes tentaram o vestibular

0,0201

Queixa na família

$5,105 \mathrm{e}^{-6}$

Sexo

0,00583

Problemas de comunicação na família

0,01044

\section{Fobia social}

Bullying

0,01747

Família rígida

0,02165

Ingresso por cotas

0,02928

Relacionamento dos pais

Bullying

$2,872 \mathrm{e}-05$

Membro com vício na família

0,006502

Família rígida

0,01274
Há mais alunos com o transtorno e que sofreram bullying

Há menos alunos com o transtorno e que sofrem de depressão

Qui quadrado

Há menos alunos com o transtorno e que mudaram de residência Há mais alunos com o transtorno no internato

Há menos alunos com o transtorno e que têm problema de comunicação na família

Mais alunos com o transtorno decidiram na adolescência
Qui

quadrado

Teste exato de

Fisher
Há mais alunos com depressão e que têm problemas de comunicação com a família

Há menos alunos com depressão cujos pais são casados

Há mais alunos que sofreram bullying e têm o transtorno do que o esperado

Teste exato de Há menos alunos com T. pânico que decidiram quando crianças

Fisher

Há mais alunos com T. pânico cujos pais são casados. Há menos cujos pais são divorciados

Wilcoxon Após Wilcoxon unilateral, concluiu-se que alunos com o

bilateral

transtorno tentam menos vezes passar para medicina

Há mais alunos com queixa na família e transtorno do pânico

Qui

quadrado

Há mais mulheres com o transtorno

Há mais alunos com problema de comunicação e transtorno
Fisher
Teste exato de

Há menos alunos que sofreram bullying e que têm fobia social

Há mais alunos com família rígida e que têm fobia social.

Há mais alunos que ingressaram por cota e têm fobia social

Há menos alunos que sofreram bullying e cujos pais são divorciados

Há mais alunos que sofreram bullying e cujos pais são casados

Há menos alunos com membros com vício na família cujos pais

Teste exato de são casados. Há mais alunos com membros com vício na família Fisher
Há mais alunos cujos pais são casados e que consideram a família rígida

Há menos alunos cujo relacionamento dos pais é "outro" e que consideram a família rígida 


\section{DISCUSSÃO}

Corroborando a literatura, foi observada nesta pesquisa a prevalência de transtornos psiquiátricos entre os estudantes de Medicina, principalmente transtornos de ansiedade, depressivos e risco de suicídio. Essas investigações apontam as mulheres como aquelas com mais transtornos psiquiátricos diagnosticados ${ }^{24}$. Em nossa pesquisa, o transtorno de pânico foi mais encontrado no sexo feminino, mas isto não aconteceu nos demais transtornos estudados. A história familiar de doença psiquiátrica vem sendo considerada fator de risco para transtornos mentais ${ }^{18,20}$. Esta situação foi observada em nossa indagação ao estabelecermos relação entre a depressão e a história familiar de algum transtorno. No rito de passagem para a academia, os estudantes sentem medo, excesso de cobrança, são frágeis e com despreparo emocional. Logo, a alta prevalência de transtornos de ansiedade generalizada, depressão e transtorno de pânico pode estar relacionada a isto. A ocorrência de mais de um transtorno no mesmo indivíduo foi incomum.

$\mathrm{O}$ risco de suicídio tem se tornado um importante item em discussão nos estudos em transtornos psiquiátricos em estudantes de Medicina. A indagação de seus possíveis gatilhos é motivo de tensão para ações de promoção à saúde. $\mathrm{O}$ bullying, a religião e os fatores protetores, como apoio familiar, são considerados ${ }^{8-10,22}$. Observamos, contudo, maior taxa de risco de suicídio nos estudantes com alguma religião, diferentemente do esperado. Foi estabelecida uma relação direta entre bullying, problemas de comunicação na família (críticas, acusações, silêncios, duplas mensagens, dificuldade em colocar-se no lugar do outro e rigidez em tentar novas formas de resolver problemas), transtornos depressivos e de pânico com o risco de suicídio. O risco de suicídio foi mais observado entre os estudantes dos primeiros períodos e entre os alunos com menos tentativas de ingresso à universidade.

Esses resultados evidenciam que muitos desses jovens são ainda adolescentes, e até mesmo os alunos com outra formação universitária são recém-saídos de um modelo escolar extremamente competitivo, voltado para o vestibular. Eles necessitam de um acolhimento diferenciado, principalmente no ciclo básico. A graduação em Medicina representa uma nova rotina de estudos, também competitiva e repleta de horas de dedicação e desafios, como o de conviver com o adoecer humano para atingir uma meta, um diploma de nível superior em saúde. Tais fatos, associados à redução do convívio familiar ou à cobrança imputada aos acadêmicos de serem excelentes, já são compreendidos como causa de sofrimento psíquico ${ }^{13,14,22-28}$.

Nesse cenário, é cabível abordar a correlação entre a mudança de moradia gerada pelo principal modelo de vestibular atual, o Exame Nacional do Ensino Médio (Enem), que permite grande mobilidade de estudantes para outras cidades e estados, e o transtorno de ansiedade generalizada. No entanto, eles estão inversamente relacionados. Levantamos a hipótese de que a interrupção do convívio familiar, embora tenha impacto negativo para a maioria, não representa um fator estressor tão relevante nesse grupo estudado. Para os demais transtornos psiquiátricos com alta prevalência - depressão, transtorno de pânico e risco de suicídio -, a mudança do local de residência não teve representação estatisticamente significativa.

O transtorno de ansiedade generalizada é maior nos últimos períodos da faculdade (internato), ou seja, nos anos de total imersão na prática médica e aumento das responsabilidades. Isto gera instabilidade psicológica. Esse é o momento do contato contínuo com as enfermidades de pacientes, com a reafirmação de limitações e frustrações, entre elas a de "curar". O modelo do cuidar ainda está no esboço do ensino, nas novas propostas de formação acadêmica ${ }^{16}$.

Foi possível observar um impacto considerável entre relacionamento dos pais e transtornos mentais dos filhos. A relação entre pais casados e transtorno de pânico foi evidenciada. Isto foi associado com a presença de famílias rígidas e com presença de alguma queixa de desconforto com a família. A relação entre depressão, pais divorciados, viúvos (mãe ou pai falecido do estudante) ou pais sem relacionamento demonstrou o impacto de um ambiente familiar segregado (convívio com mau relacionamento com os pais). Não podemos esquecer que o impacto da morte de um dos pais sobre a psicodinâmica de um jovem adulto é relevante. E que, muitas vezes, esse é um momento no ciclo vital desses jovens, a perda dos avós.

A fobia social, diferentemente do esperado, demonstrou menor associação com a presença de famílias rígidas, contudo mostrou relação com o bullying (na família ou na escola). Ela foi relacionada, por meio de ações afirmativas, ao ingresso na universidade. A hipótese é a de que os dois últimos gerariam baixa autoestima e, assim, o receio de estabelecer relacionamentos sociais. $\mathrm{O}$ ingresso por meio de cotas raciais ou de renda pode gerar desconforto e discriminação no ambiente da faculdade. Os estudantes cotistas são, muitas vezes, apreciados como não merecedores da vaga na universidade. Assim, uma ação que seria favorável à estruturação de um indivíduo torna-se desqualificada.

O estudo já considerou que a configuração da dinâmica familiar está relacionada à formação da saúde mental dos membros dela ${ }^{17,18,20}$ e a predisposição a transtornos psiquiátricos, nessa falta. Problemas de comunicação na família, depressão e risco de suicídio colaboram para elucidar essa questão. Em nosso estudo, entretanto, certas variáveis condizentes com dis- 
funções familiares, como ter parente violento ou com vício, não tiveram relação estatística significativa com os transtornos aqui pesquisados, mas podemos inferir que tal resultado se deva ao número reduzido de voluntários com esse perfil familiar.

Reiterando o que já demonstramos de outra maneira, a ansiedade foi menor para os estudantes de Medicina com problemas no relacionamento com a família. Assim, tal como foi observado na mudança de residência (moradia), o transtorno de ansiedade generalizada parece estar mais relacionado à própria formação médica e a suas dificuldades do que às dinâmicas familiares.

É possível inferir a importância do relacionamento familiar como futuro alicerce para as demais relações sociais a serem estabelecidas na vida dos jovens. Um ambiente familiar disfuncional poderia criar dificuldade na construção de novos relacionamentos, entre eles o de ser um bom comunicador com a família do paciente e um bom médico de família ${ }^{17,18,20,21}$.

Os resultados desta pesquisa têm como meta a cooperação para se pensar, segundo as novas diretrizes curriculares dos cursos de saúde, como se encontra a saúde mental dos estudantes numa instituição federal brasileira e se as novas configurações familiares contribuem para o adoecimento psíquico desses adultos jovens.

\section{CONCLUSÕES}

Os dados colhidos demonstraram correlações entre disfunções familiares e transtornos psiquiátricos, como depressão, risco de suicídio, transtorno de pânico e transtorno de ansiedade generalizada. A formação médica, a da saúde, é um processo repleto de desafios intelectuais, éticos, emocionais ou pessoais, além do conteúdo biomédico, pois tanto o profissional como o estudante, ao entrarem em contato com seu paciente, família e suas próprias limitações, se deparam com sua própria vida, suas frustrações, seus conflitos pessoais e familiares.

As socializações primárias em ambiente familiar disfuncional revelam transtornos mentais/suicídio durante a socialização secundária (o curso de Medicina). Com base em nosso estudo, verificamos que são necessários dispositivos para capacitar o grupo da "família acadêmica" para que os alunos questionem a dinâmica da família original e o ambiente escolar seja um novo objeto de identificação. Tornam-se importantes as relações dialógicas entre tutores, mentores, preceptores, docentes, residentes e discentes, no espaço transicional e potencial do ambiente de ensino-aprendizagem, com espaços e cenários em que educadores médicos identifiquem precocemente o sofrimento psíquico do futuro médico.

A área das Humanidades em Medicina favoreceria em muito o desenvolvimento psicoemocional desses jovens estu- dantes, pois ela é responsável por apresentar situações cotidianas, que extrapolam a esfera da biomedicina. Entretanto, ainda é um núcleo em desenvolvimento, pois a mudança do modelo flexneriano para o da integralidade encontra resistências. A medicina ainda não é vista como uma ciência humana, estando o futuro médico submetido a uma antiga ideologia, que valoriza o campo biomédico.

Essa situação encontra-se em desacordo com a literatura sobre o tema. A formação médica segue fragmentada. Ao longo do curso, o aluno aprende processos psicofisiopatológicos de doença, sinais e sintomas, síndromes, formas de tratamento, mas não aprende a reabilitar indivíduos após o adoecimento. Logo, ao não ser capacitado para lidar com o seu paciente como um "ser" dotado de sentimentos, anseios e frustrações, com problemas sociais e familiares inerentes, ele não aprende a se conhecer e a procurar ajuda no caso de uma situação de identificação no campo da prática. Ou seja, a cuidar de si e das necessidades da população. E, ao não encontrar um ambiente que compreenda as questões aqui apresentadas, poderá desenvolver outros transtornos, como estresse, somatização e burnout (CID -10).

\section{REFERÊNCIAS}

1. Ellenberger HF. El descobrimento do inconsciente. Madrid: Editorial Gredos; 1976.

2. Rezende JM. O juramento de Hipócrates. In: Rezende JM. À sombra do plátano: crônicas de história da medicina. São Paulo: Editora Unifesp; 2009. p. 31-48.

3. Millan LR, Arruda PCV. Assistência psicológica ao estudante de medicina: 21 anos de experiência. RevAssocMedBras [Internet]. 2008 [citado 2019 Abr 28];54(1):90-4. Disponível em: http:/ / www.scielo.br/pdf/ramb/v54n1/27.pdf

4. Millan LR, De Marco OLN, Rossi E, Arruda PCV. O universo psicológico do futuro médico. São Paulo: Casa do Psicólogo; 1999.

5. Nunes TC. Investigação sobre queixas de memória e transtornos associados em acadêmicos de medicina da Universidade Federal Fluminense [dissertação]. Niterói: Universidade Federal Fluminense; 2017

6. Aragão JCS, Casiraghi B, Mota ÉM, Abrahão MAB, de Almeida TA, Baylão ACP, et al. Saúde mental em estudantes de medicina. RevEstudInvestPsicol Educ. 2017;38-41.

7. Bento LMA, de Andrade LP, Sales A, de Souza AP, de Souza AFP, Batistona GT, et al. Percepção dos alunos de medicina quanto a aprendizagem $X$ ansiedade na metodologia ativa. Revi EnsEducCienc Hum. 2017;18(2):178-82.

8. Franken I, Gonçalves C, Costa A, Araújo V, Sousa B. O sofrimento psíquico na graduação de médicos. In: Leal I, von 
Humboldt S, Ramos C, Valente AF, Ribeiro JLP. Actas do $12^{\circ}$ Congresso Nacional de Psicologia da Saúde [Internet]. Lisboa: ISPA - Instituto Universitário; 2018 [citado 2019 Abr 29]. p. 385-403.

9. Fiorotti KP, Rossoni RR, Borges LH, Miranda AE. Transtornos mentais comuns entre os estudantes do curso de medicina: prevalência e fatores associados. J Bras Psiquiatr. 2010;59(1):17-23.

10. Marafanti I, D’Elia G, Pinheiro MCP, Cordeiro Q, Alves TCTF. Influência de sintomas ansiosos no desempenho acadêmico de formandos de medicina. ArqMedHospFacCiencMed Santa Casa São Paulo. 2013;58:18-23.

11. Campos TD, Silveira RD, Neves CP, Dal'Col PP, Dias PEB, Ribeiro FC, et al. Formação em saúde mental no curso de medicina: a experiência do SAMU. Sinapse Múltipla. 2017;6(2):252-6

12. Cunha AF, Santos VKR. Avaliação de transtornos mentais comuns em estudantes de medicina do campus lagarto: prevalência e fatores associados [monografia]. Lagarto: Universidade Federal de Sergipe; 2018.

13. Rocha ES, Sassi AP. Transtornos mentais menores entre estudantes de medicina. RevBrasEduc Med. 2013;37(2):210-6.

14. Amorim BB, Moraes L, Sá ICG, Silva BBG, Camara Filho JWS. Saúde mental do estudante de medicina psicopatologia, estresse, sono e qualidade de vida. RevPsi Divers Saúde [Internet]. 2018 [citado 2019 Abr 20];7(2):245-54. Disponível em: https://www5.bahiana.edu.br/index.php/ psicologia/article/view/1911/1829

15. Ministério da Educação (BR). Resolução no 3, de 20 de junho de 2014. Institui Diretrizes Curriculares Nacionais do Curso de Graduação em Medicina e dá outras providências. Diário Oficial da União: República Federativa do Brasil. 2014 Jun 23. Seção 1:8-11.

16. Franco CAGS, Cobasi MR, Franco RS. Currículo de medicina e as competências propostas pelas diretrizes curriculares. RevBrasEduc Med. 2014;38(2)221-30.

17. Souza MS, Baptista MN, Alves GAS. Suporte familiar e saúde mental: evidência de validade baseada na relação entre variáveis. Aletheia. 2008;28:32-44.

18. Amaral M. Estrutura e dinâmica familiar; famílias funcionais e disfuncionais [Internet]. 2015 [citado 2018 Out 20]. Disponível em: https://ares. unasus.gov.br/acervo/handle/ARES/21521

19. Berger PL, Luckmann T. A construção social da realidade: tratado de sociologia do conhecimento. Petrópolis: Vozes; 1976.

20. Souza MS, Baptista MN. Associações entre suporte familiar e saúde mental. PsicolArgum. 2008;26(54)207-15.
21. Gomes JV. Família e socialização. Psicol USP. 1992;3(1/2):93105.

22. Katsurayama M, Becker MAA, Gomes NM, Santos MC, Makimoto FH, Santana LLO. Fatores de risco e proteção em estudantes de medicina da Universidade Federal do Amazonas. PsicolAmLat [Internet]. 2009 [citado 2018 Out 15];16. Disponível em: http://pepsic.bvsalud.org/scielo. php?script=sci_arttext\&pid=S1870-350X2009000100006

23. Amorim P. Mini InternationalNeuropsychiatric Interview (MINI): validação de entrevista breve para diagnóstico de transtornos mentais. RevBras Psiquiatr. 2000;22(3)106-15.

24. Ferreira RAR, Parrão JA. Transtornos mentais na mulher e as possibilidades de intervenção do assistente social: um estudo na ubs "belo horizonte" de presidente prudente. Intertemas. 2015;9(9):1-24.

25. Medeiros PP, Bittencourt FO. Fatores associados à ansiedade em estudantes de uma faculdade particular. Id OnLineRevMult Psicol. 2017;10(33):42-55.

26. Santa ND, Cantilino A. Suicídio entre médicos e estudantes de medicina: revisão de Literatura. RevBrasEduc Med. 2016;40(4)772-80.

27. Costa EFO, Santana YS, Santos ATRA, Martins LAN, Melo EV, Andrade TM. Sintomas depressivos entre internos de medicina em uma universidade pública brasileira. RevAssocMed Bras. 2012;58(1):53-9.

28. Leal SDP, Salgado DR, Mello DRB. Os estudantes de medicina e os aspectos emocionais envolvidos nesse processo. REINPEC. 2017;3(2):178-91.

29. Nóbrega-Therrien SM, Souza PMM, Pinheiro FMC, Castro VS. Formação para a Estratégia Saúde da Família na Graduação em Medicina. RevBrasEduc Med. 2015;39(1):112-8.

\section{Agências de Fomento:}

Universidade Federal do Estado do Rio de Janeiro (Unirio).

Número do projeto cadastrado na Plataforma Brasil: 67590317.5.0000.5258.

Número do parecer de aprovação do projeto pelo Comitê de Ética e Pesquisa Institucional: 2.185.509 no dia 25 de julho de 2017.

\section{CONTRIBUIÇÃO DOS AUTORES}

Isabelle Christine de Moraes Motta trabalhou em todas as fases do projeto desde a concepção do estudo, coleta, análise e interpretação dos dados; redação do artigo e aprovação da versão a ser publicada.

Rita de Cássia Menezes Soares trabalhou em todas as fases do estudo e contribuiu na organização dos dados, na análise e interpretação dos dados para tabulação estatística. 
Terezinha de Souza Agra Belmonte trabalhou na concepção do estudo, na análise e interpretação dos dados e na revisão crítica e aprovação da versão a ser publicada.

\section{CONFLITO DE INTERESSES}

Todos os autores do manuscrito afirmam que não têm qualquer conflito de interesse com o tema abordado no artigo, itens citados. Declaram que este artigo é original e que o trabalho, em parte ou na íntegra, ou qualquer outro trabalho com conteúdo substancialmente similar, não será enviado a outro periódico científico enquanto sua publicação estiver sendo considerada pela Revista Brasileira de Educação Médica.

\section{ENDEREÇO PARA CORRESPONDÊNCIA}

Universidade Federal do Estado do Rio de Janeiro (Unirio) Centro de Ciências Biológicas e da Saúde (CCBS)

Escola de Medicina e Cirurgia do Rio de Janeiro

Rua Silva Ramos, 32 - Tijuca

CEP 20270-330 - RJ

E-mail: isabellec.motta@yahoo.com.br 